\title{
ĐỀ XUẤT QUY TRÌNH PHÂN LOẠI ẢNH VÊ TINH DỰA TRÊN GIẢI PHÁP NÂNG CAO Độ CHÍNH XÁC CỦA CÔNG TÁC PHÂN LOẠI ẢNH KHU VỰC CÓ LỚP PHỦ HỖN HỘP
}

\author{
PHAM MINH HẢl \\ Viện Khoa học Đo đạc và Bản đồ
}

\section{Tóm tắt:}

Ngày nay, các ứng dụng ảnh vệ tinh phục vụ cho các mục đích nghiên cứu, thí nghiệm hay ứng dụng phục vụ đời sống xã hội nói chung và lĩnh vực Tài Nguyên Môi Trường nói riêng ngày càng trở nên phổ biến. Quá trình chiết tách thông tin được sử dụng phổ biến là các phương pháp phân loại ảnh có kiểm định và không có kiểm định. Phân loại có kiểm định là một phương pháp xác suất có khả năng sắp xếp những điểm ảnh do người sử dụng định nghĩa thành những lớp khác nhau. Tuy nhiên, khi thực hiện phân loại ảnh có kiểm định ở khu vực có bề mặt lớp phủ hỗn hợp, độ chính xác của kết quả phân loại ảnh không cao do các kết quả phân loại ảnh bị ảnh hưởng bởi vấn đề nhiễu điểm ảnh. Nhiễu điểm ảnh là hiện tượng xảy ra khi một điểm ảnh có giá trị điểm ảnh thuộc lớp $A$ nhưng trong kết quả phân loại ảnh thì điểm ảnh đó sẽ được phân loại vào lớp khác ngoài lớp $A$. Trong các úng dụng sử dụng tư liệu ảnh vệ tinh độ phân giải vì̛a và nhỏ, bề mặt đất hỗn hợp bao gồm nhiều đối tượng ảnh, công tác xử lý vấn đề nhiễu điểm ảnh bằng việc can thiệp vào các thành phần đất, nước, thực vật trên mỗi điểm ảnh nhằm cải thiện độ chính xác của kết quả phân loại là có tính cấp thiết cao. Trên số báo trước của Tạp chí Khoa học Đo đạc và Bản đồ, nhóm nghiên cứu đã trình bày phần cơ sở khoa học của giải pháp kỹ thuật nâng cao độ chính xác của công tác phân loại ảnh khu vực có lớp phủ hỗn hợp. Trong số báo này, nhóm nghiên cứu sẽ trình bày Quy trình phân loại dụva trên giải pháp nâng cao độ chính xác của công tác phân loại ảnh dựa theo giá trị phổ thực và tỷ lệ thành phần đất, nước, thực vật.

\section{Mỏ̉ đầu}

Trong lĩnh vực giám sát Tài nguyên và Môi trường, phương pháp phân loại ảnh được sử dụng để khai thác dữ liệu ảnh vệ tinh. Quá trình tách thông tin từ ảnh vệ tinh có thể được thực hiện bằng các phương pháp phân loại ảnh. Hai phương pháp phân loại ảnh thông dụng hiện nay là phương pháp phân loại không kiểm định và phương pháp phân loại có kiểm định. Trong phạm vi nghiên cứu này, đối tượng nghiên cứu để nâng cao độ chính xác là phương pháp phân loại có kiểm định.
Bề mặt đất được ghi lại bởi các điểm ảnh, mỗi điểm ảnh thường chứa nhiều hơn một loại lớp phủ. Nhiễu điểm ảnh là hiện tượng xảy ra khi một điểm ảnh có giá trị điểm ảnh thuộc lớp $A$ nhưng trong kết quả phân loại ảnh thì điểm ảnh đó sẽ được phân loại vào lớp khác ngoài lớp $A$. Ba đối tượng đặc trưng được mô tả tồn tại trong mỗi điểm ảnh đó là: Nước-Đất-Thực vật. Mỗi đối tượng này sẽ chiếm tỷ lệ nhất định trong mỗi điểm ảnh. Ví dụ, nếu một điểm ảnh có tỷ lệ Nước: $50 \%$, Đất:30\%, Thực vật:20\% thì điểm ảnh này sẽ thuộc lớp Nước do đối

Ngày nhận bài: 24/5/2016, ngày chuyển phản biện: 27/5/2016, ngày chấp nhận phản biện: 03/6/2016, ngày chấp nhận đăng: 06/6/2016 
tượng nước chiếm tỷ lệ cao nhất trong điểm ảnh. Độ chính xác công tác phân loại ảnh bị chi phối bởi hiện tượng nhiễu điểm ảnh sau phân loại ảnh. Tuy nhiên, cho tới nay tại nước ta các nghiên cứu về giảm nhiễu điểm ảnh chưa quan tâm nhiều, phát triển giải pháp nâng cao độ chính xác kết quả phân loại ảnh có tính cấp thiết cao, nâng cao hiệu quả khai thác dữ liệu viễn thám. Trên cơ' sở' đánh giá tình hình thực tế, đề tài: "Nghiên cứu giải pháp kỹ thuật nâng cao độ chính xác của công tác phân loại ảnh khu vực có lớp phủ hỗn hợp" đã được thực hiện tại Viện Khoa học Đo đạc và Bản đồ, Bộ Tài nguyên và Môi trường.

Trên số báo trước của Tạp chí Khoa học Đo đạc và Bản đồ, nhóm nghiên cứu đã trình bày phần cơ sở khoa học của giải pháp kỹ thuật nâng cao độ chính xác của công tác phân loại ảnh khu vực có lớp phủ hỗn hợp. Trong số báo này, nhóm nghiên cứu sẽ trình bày Quy trình phân loại dựa trên giải pháp nâng cao độ chính xác của công tác phân loại ảnh dựa theo giá trị phổ thực và tỷ lệ thành phần đất, nước, thực vật.

2. Quy trình phân loại dựa trên giải pháp nâng cao độ chính xác của công tác phân loại ảnh dựa theo giá trị phổ thực và tỷ lệ thành phần đất, nước, thực vật

Quy trình phân loại ảnh dựa trên giải pháp nâng cao độ chính xác của công tác phân loại ảnh dựa theo giá trị phổ và tỷ lệ thành phần đất, nước, thực vật được nhóm nghiên cứu phát triển trên cơ sở kết hợp phương pháp phân loại ảnh có kiểm đinh truyền thống và phương pháp phân loại ảnh sử dụng thành phần 3 đối tượng đất, nước, thực vật trên ảnh. (Xem hình 1)

\subsection{Thông tin dữ liệu thực nghiệm}

Tác giả tiến hành thử nghiệm ở các độ phân giải trung bình ASTER $(15 \mathrm{~m})$ với tính chất ở 2 khu vực có lớp phủ phức tạp là khu

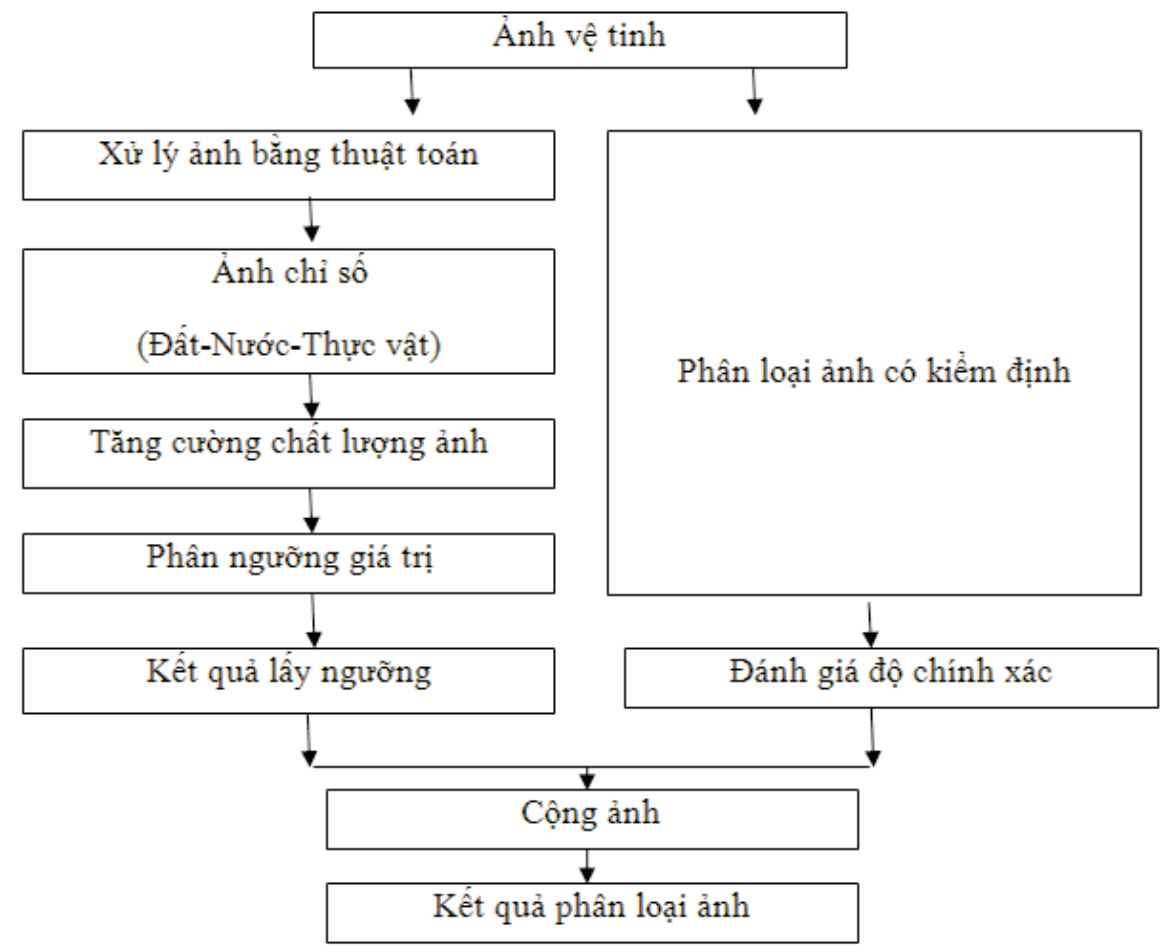

Hình 1: Quy trình phân loại ảnh 
vực đô thị có cả đối tượng thực phủ, đất, nước. Ảnh có kích thước $1413 \times 1414$ pixel, 14 kênh phổ. (Xem hình 2)

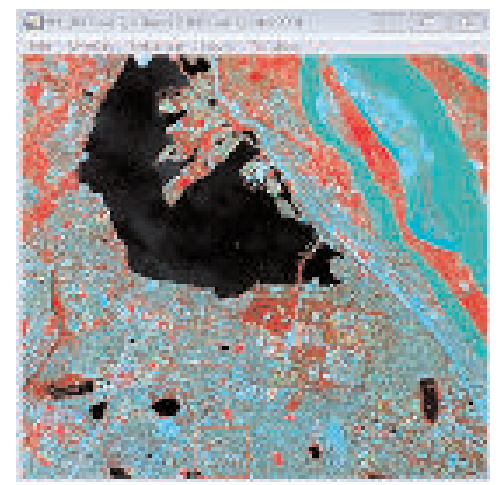

Hình 2: Ảnh ASTER khu vực Hà Nội tổ hợp màu giả lục-đỏ-cận hồng ngoại

\subsection{Phân loại ảnh có kiểm định}

Tác giả sử dụng phương pháp phân loại có kiểm định Maximum Likelihood để phân loại ảnh ASTER khu vực thực nghiệm. Quá trình giải đoán ảnh dựa trên sự kết hợp giữa dữ liệu ảnh vệ tinh và kết quả khảo sát thực địa nhằm đưa ra bộ mẫu. Công tác lấy mẫu được tiến hành trên ảnh với 6 mẫu là: nước, thực vật, đô thị, đất ẩm, đường xá, đất trống. (Xem hình 3)

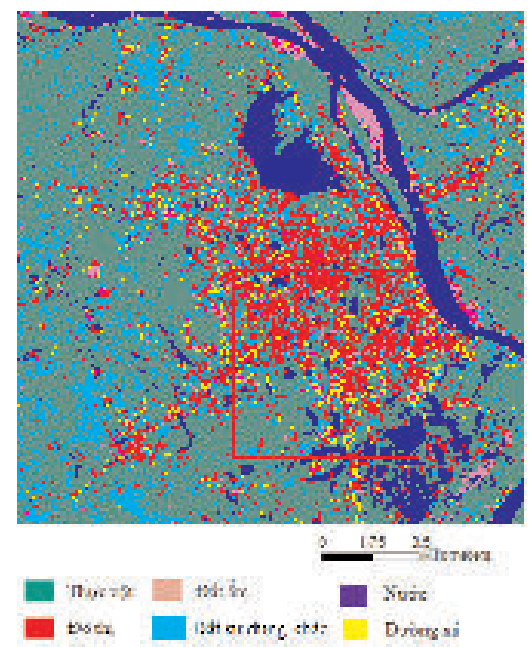

Hình 3: Kết quả phân loại ảnh bằng phương pháp phân loại ảnh có kiểm định Maximum Likelihood
2.3. Thực nghiệm tính toán xác định giá trị phổ thực của 3 đối tượng đất, nước, thực vật trên ảnh

Để chiết tách các đối tượng trên ảnh, việc xác định các giá trị phổ thực của các đối tượng đóng vai trò quan trọng đặc biệt là vai trò của 3 yếu tố chính trên ảnh đó là đất, nước, và thực vật. Quá trình phân tách đối tượng trong từng điểm ảnh là quá trình tính toán phân đoạn tham gia của các giá trị phổ sơ cấp trong điểm ảnh. Tác giả đã xây dựng môđun tính toán giá trị phổ thực bằng ngôn ngữ lập trình $\mathrm{C \#}$. Sau khi chạy chương trình, các kết quả chương trình sẽ thể hiện các giá trị phổ thực của ba đối tượng đất, nước, thực vật như sau:

Giá trị phổ thực của thực vật là 76 (trục x) Giá trị phổ thực của đất là 110 (trục y) Giá trị phổ thực của nước là 76 (trục $x$ )

Từ kết quả tính toán giá trị phổ thực trên ta có thể xây dựng được một tam giá phổ với 3 đỉnh là 3 đối tượng đất, nước, thực vật (hình 4). Trong nghiên cứu này, nhóm nghiên cứu sẽ tiến hành tính toán với các điểm ảnh nằm trong tam giác phổ này, đối với các điểm ảnh nằm ngoài tam giác phổ được định nghĩa là các điểm ảnh nhiễu và bị loại.

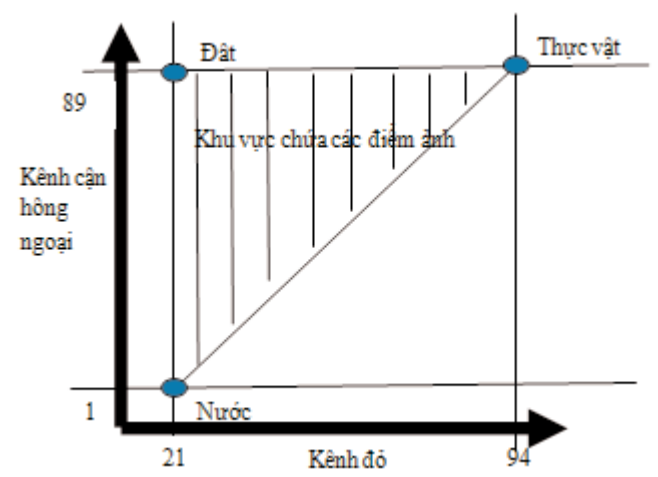

Hình 4: Hình thành tam giác phổ với 3 đỉnh

là 3 đối tượng đất, nước, thực vật trong không gian 2 chiều với trục $x$ là kênh đỏ và trục y là kênh Cận hồng ngoại 
2.4. Thực nghiệm tính toán tỷ lệ thành phần 3 đối tượng đất, nước, thực vật

Sau khi tính toán các giá trị endmember của 3 yếu tố đất, nước, thực vật trên ảnh thực nghiệm, nhóm nghiên cứu đã xác định được tam giác phổ với 3 đỉnh là 3 đối tượng đất, nước, và thực vật. Nội dung phần này sẽ đề cập tới công tác tính toán tỷ lệ của 3 đối tượng đất, nước, và thực vật trên 1 điểm ảnh theo các công thức toán học được mô tả trong Tạp chí Khoa học Đo đạc và Bản đồ số 28/6/2016. Nhóm nghiên cứu đã xây dựng môđun tính toán tỷ lệ thành phần 3 đối tượng đất, nước, thực vật bằng ngôn ngữ lập trình C\#. Sau khi chạy chương trình, các kết quả chương trình sẽ thể hiện ba ảnh chỉ số: chỉ số đất, chỉ số nước, chỉ số thực vật (hình 5).

2.5. Phân loại ảnh sử dụng quy trình nâng cao độ chính xác của công tác phân loại ảnh dựa theo giá trị phổ và tỷ lệ thành phần đất, nước, thực vật

Sau khi có kết quả chiết tách đối tượng thực vật và nước từ ảnh chỉ số thực vật và ảnh chỉ số nước, nhóm nghiên cứu tiến hành cộng ảnh thay thế các đối tượng đất và nước trên ảnh phân loại bằng phương pháp phân loại có kiểm định được thực hiện ở phần 2.2 với các đối tượng thực vật và nước vừa được chiết tách. Phương pháp tiếp theo được sử dụng là phương pháp cộng ảnh thay thế các khu vực thực vật và nước được lấy ngưỡng với kết quả phân loại có kiểm định. Tác giả đã xây dựng mô đun cộng ảnh bằng ngôn ngữ lập trình $\mathrm{C \#}$. Sau khi chạy chương trình, kết quả phân loại ảnh được thể hiện trên hình 6.

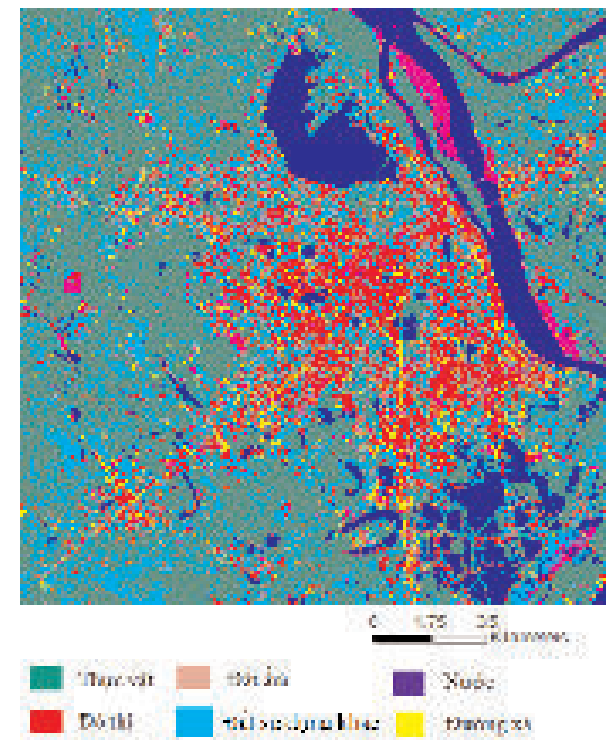

Hình 6: Kết quả phân loại ảnh sử dụng giải pháp nâng cao độ chính xác của công tác phân loại ảnh dựa theo giá trị phổ và tỷ lệ thành phần đất, nước, thực vật

2.6. Đánh giá độ kết quả phân loại ảnh sử dụng giải pháp nâng cao độ chính xác của công tác phân loại ảnh dựa theo giá trị phổ và tỷ lệ thành phần đất, nước, thực vật

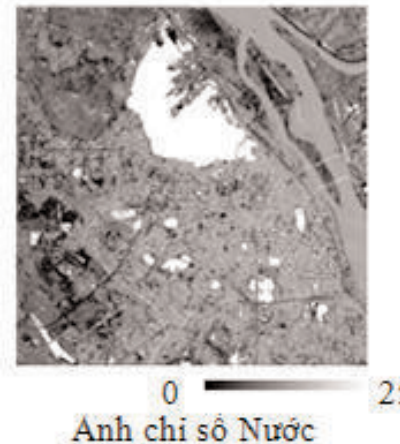

Anh chi số Nước

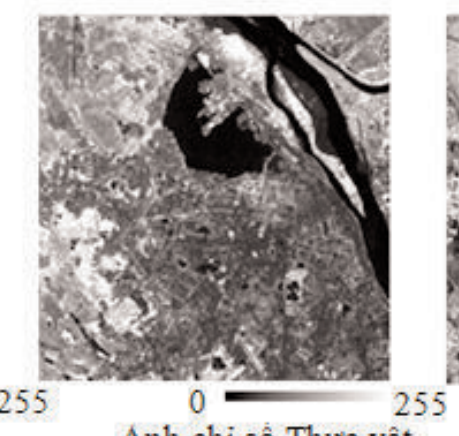

Anh chi sồ Thực vật

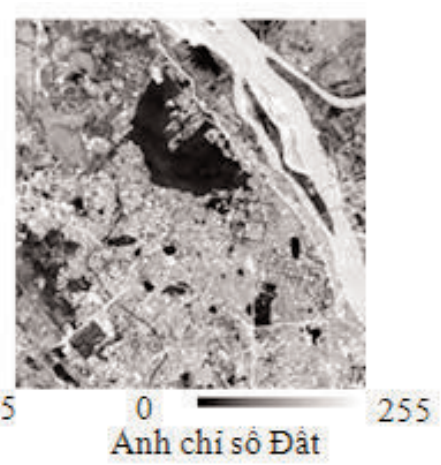

Anh chi sồ Đât

Hình 5: Các ảnh chỉ số Nước, Đất, và Thực vật từ kết quả tính toán tỷ lệ thành phần đất, nước, thực vật trên ảnh 
Công tác đánh giá độ chính xác kết quả của nghiên cứu được thực hiện bởi hai phương pháp là: 1 . Phương pháp lấy mẫu so sánh giữa kết quả chiết tách nước, thực vật, kết quả phân loại ảnh có kiểm định và ảnh vệ tinh gốc; 2. Phương pháp thống kê số điểm ảnh và diện tích khu vực thực phủ và mặt nước của kết quả chiết tách nước, thực vật, kết quả phân loại ảnh có kiểm định. Với phương pháp lấy mẫu so sánh, 10 khu vực sẽ được lấy mẫu để tiến hành công tác đánh giá so sánh, trong đó 5 mẫu là đánh giá độ chính xác kết quả chiết tách thực vật và 5 mẫu để đánh giá độ chính xác kết quả chiết tách bề mặt nước. Với phương pháp thống kê, nhóm nghiên cứu tiến hành tính so sánh số lượng điểm ảnh và diện tích của kết quả chiết tách thực vật và nước và kết quả phân loại ảnh có kiểm định. Tác giả đã xây dựng chương trình tính toán điểm ảnh và diện tích bằng ngôn ngữ lập trình $\mathrm{C \#}$. Sau khi chạy chương trình, kết quả phân loại ảnh được thể hiện trên các hình dưới đây.

Phương pháp lấy mẫu so sánh giữa kết quả chiết tách nước, thực vật, kết quả phân loại ảnh có kiểm định và ảnh vệ tinh gốc

Nhóm nghiên cứu tiến hành đánh giá độ chính xác của đối tượng thực vật và nước ở trên ảnh. Do vậy, sản phẩm của nghiên cứu sẽ được thể hiện còn 2 đối tượng là nước và thực vật. Dữ liệu phục vụ công tác đánh giá độ chính xác tại đây được mô tả trên hình 7 .

10 khu vực trên ảnh được lựa chọn để đánh giá độ chính xác ảnh chiết tách thực vật và nước (bảng 1). Kết quả thực hiện bởi nghiên cứu này cho thấy có độ chính xác cao hơn so với kết quả của phân loại đối tượng trên ảnh phân loại sử dụng phương pháp phân loại có kiểm định Maximum Likelihood. Nhìn vào các mẫu được thử nghiệm, chúng ta có thể thấy độ chính xác kết quả phân loại theo phương pháp phân loại có kiểm định dựa vào độ chính xác kết quả lấy mẫu. Trong phạm vi bài báo này, nhóm nghiên cứu sử dụng phương pháp phân loại có kiểm định Maximum Likelihood. Với các mẫu, việc lựa chọn mẫu phụ thuộc vào kinh nghiệm và tính chủ quan của người lấy mẫu. Ví dụ, tại một số khu vực ruộng lúa hay khu vực trồng hoa màu, sau khi thu hoạch thì cỏ xuất hiện. Đối với đối tượng cỏ, do lượng chrolophyl hấp thụ yếu hơn thực vật nói chung trên ảnh nên trên ảnh tổ hợp màu giả có màu hồng. Với khu vực có ít cỏ trên nhiều diện tích đất trống hơn, việc lấy mẫu cả những đối tượng này sẽ làm cho kết quả phân loại bị nhiễu, vì hiện tượng nhiễu điểm ảnh sẽ xảy ra do mẫu thực vật (cỏ) với mẫu đất. Độ chính xác của kết quả phân loại ảnh thực hiện bằng phương pháp phân loại có kiểm định sẽ bị

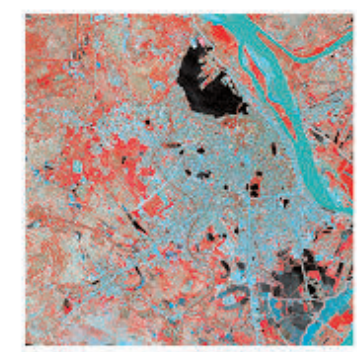

Ảnh ASTER tồ hợp màu giả Lục-Đỏ-Cận hồng ngoại

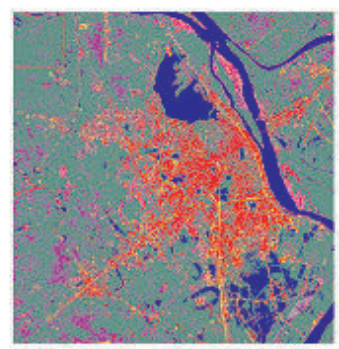

Kết quả phân loại ảnh có kiềm định

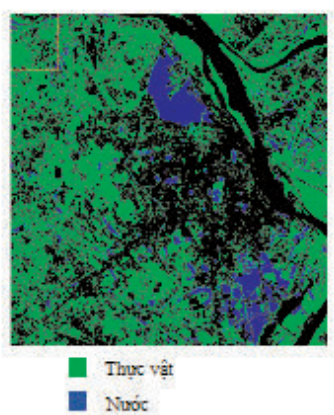

Kết quả chiết tách nước, thực vật

Hình 7: Dữ liệu phục vụ công tác đánh giá độ chính xác của kết quả nghiên cứu 
ảnh hưởng vì trên kết quả phân loại ảnh có kiểm định là lớp thực vật thay vì lớp đất trống như trên thực địa. Kết quả hiển thị trên Bảng 1 cho thấy các nhiều diện tích khu vực đất trống được phân loại thành lớp thực vật. Hiện tượng nhiễu ảnh do phân loại nhầm lớp giữa lớp nước và đất ẩm cũng diễn ra với cùng cơ chế như trên trong ảnh phân loại có kiểm định.
Đối với kết quả phân loại được thực hiện bằng phương pháp luận của nghiên cứu thì hiện tượng nhiễu điểm ảnh đã được giảm. Do không chịu sự chi phối của công tác lấy mẫu nên trên kết quả phân loại ảnh các đối tượng được thể hiện rõ nét, ít có hiện tượng sai số phân loại do nhầm lớp như ở kết quả phân loại có kiểm định.

Bảng 1: Đánh giá độ chính xác kết quả phân loại ảnh

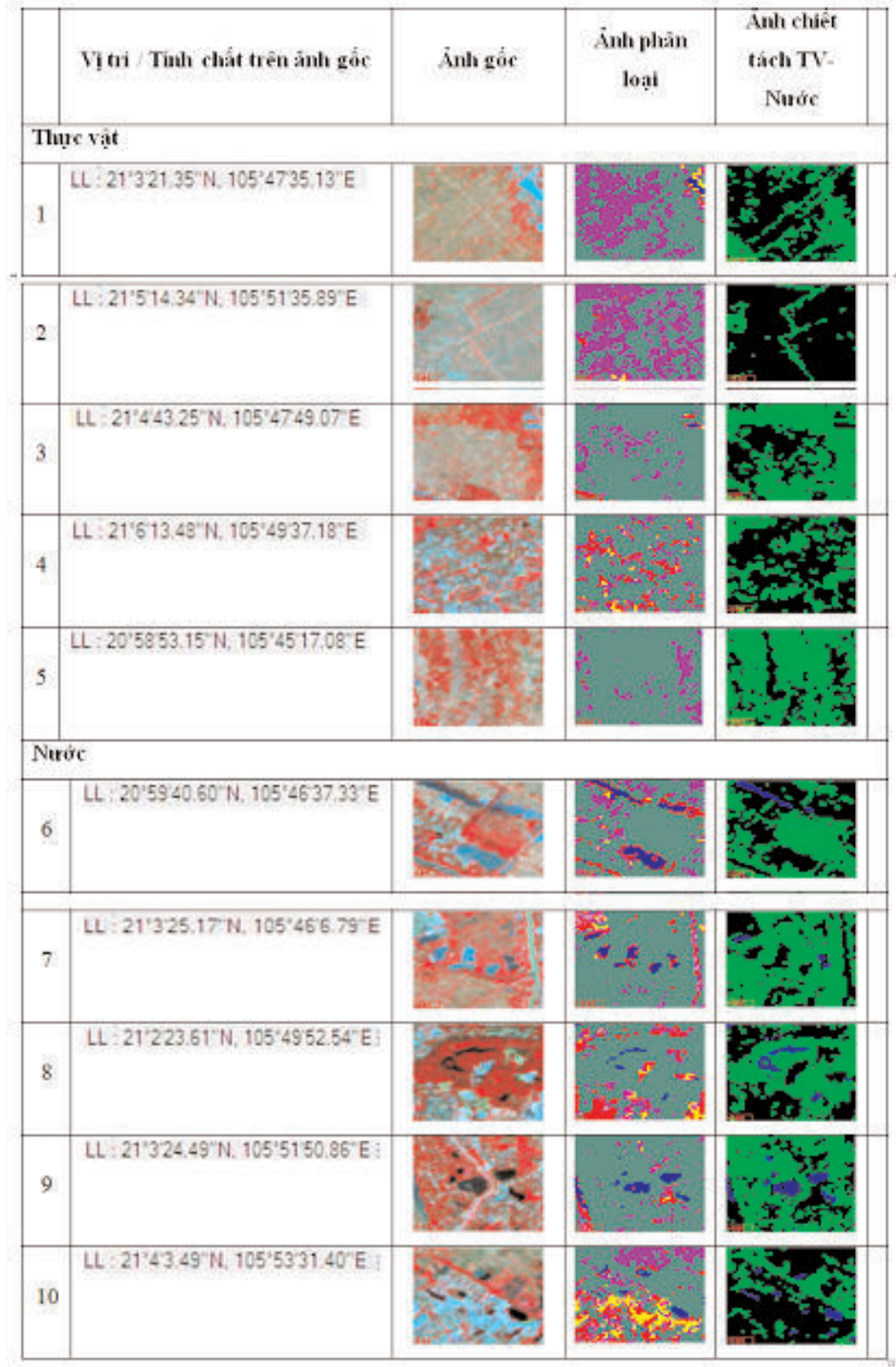


Phương pháp so sánh diện tích giữa kết quả phân loại được thực hiện bởi phương pháp luận của bài báo và kết quả phân loại

Sau khi thực hiện phương pháp so sánh đối chiếu đánh giá độ chính xác ở trên, nhóm nghiên cứu tiến hành so sánh diện tích và số điểm ảnh đối tượng thực vật và nước trên kết quả phân loại được thực hiện bởi phương pháp luận của nhóm nghiên cứu và kết quả phân loại. Kết quả tính toán số lượng điểm ảnh của đối tượng thực vật và nước thể hiện như sau:

Bảng 2: Số lượng điểm ảnh của đối tượng nước và thực vật trên hai kết quả phân loại ảnh

\begin{tabular}{|c|c|c|c|}
\hline $\begin{array}{c}\text { Số lượng } \\
\text { điểm ảnh }\end{array}$ & $\begin{array}{c}\text { Phân loại có } \\
\text { kiếm định }\end{array}$ & $\begin{array}{c}\text { Phân loại sử } \\
\text { dụng tỷ lề } \\
\text { thành phần } \\
\text { đất, nước, } \\
\text { thực vật }\end{array}$ & Sai số \\
\hline Nước & 95096 & 86649 & 8447 \\
\hline Thực vật & 893871 & 663138 & 230733 \\
\hline
\end{tabular}

Xu hướng giảm điểm ảnh của đối tượng nước và thực vật giữa kết quả phân loại có kiểm định và kết quả phân loại sử dụng tỷ lệ thành phần đất, nước, thực vật được thể hiện trên đồ thị sau:

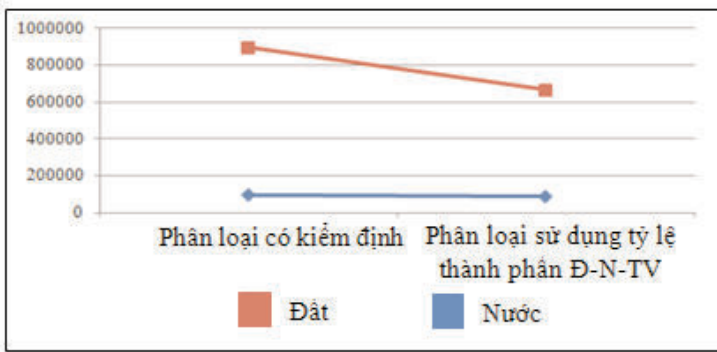

Hình 8: Đồ thị thế hiện so sánh số lượng điểm ảnh giữa kết quả phân loại có kiểm định và kết quả phân loại sử dụng tỷ lệ thành phần đất, nước, thực vật

Kết quả so sánh điểm ảnh và diện tích giữa kết quả phân loại có kiểm định và kết quả phân loại sử dụng tỷ lệ thành phần đất, nước, thực vật cho thấy số lượng điểm ảnh và diện tích của kết quả phân loại sử dụng tỷ lệ thành phần đất, nước, thực vật giảm nhiều so với số lượng điểm ảnh và diện tích của kết quả phân loại sử dụng phương pháp phân loại có kiểm định. Đặc biệt với đối tượng thực vật, số lượng điểm ảnh trên kết quả phân loại sử dụng tỷ lệ thành phần đất, nước, thực vật giảm $1 / 3$ so với số lượng điểm ảnh trên kết quả phân loại sử dụng phương pháp phân loại có kiểm định. Số lượng điểm ảnh bị nhiễu trong khu vực thử nghiệm chủ yếu tập trung vào nhiễu thực vật sang đối tượng khác như nhiễu thực vật-đất, thực vật-nước.

\section{Kết luận}

Nghiên cứu đã góp phần giải quyết những vấn đề liên quan đến giảm nhiễu của các đối tượng trên ảnh đồng thời nâng cao chính xác kết quả phân loại ảnh. Kết quả của nghiên cứu được thể hiện ở hai sản phẩm đó là: Giải pháp nâng cao độ chính xác của công tác phân loại ảnh khu vực có lớp phủ hỗn hợp và Quy trình phân loại ảnh dựa trên giải pháp nâng cao độ chính xác của công tác phân loại ảnh dựa theo giá trị phổ và tỷ lệ thành phần đất, nước, thực vật. Với giải pháp nâng cao độ chính xác của công tác phân loại ảnh khu vực có lớp phủ hỗn hợp, nhóm nghiên cứu đã phát triển được một phương pháp giảm nhiễu điểm ảnh bằng cách tính toán giá trị phổ thực của 3 yếu tố đất, nước, thực vật và tỷ lệ thành phần của các yếu tố này trên ảnh. Nghiên cứu đã đề xuất được Quy trình phân loại ảnh dựa trên giải pháp nâng cao độ chính xác của công tác phân loại ảnh dựa theo giá trị phổ và tỷ lệ thành phần đất, nước, thực vật. Kết quả phân loại thể hiện khu vực phân bố của ba đối tượng đất, nước, thực vật rõ nét, và có độ chính xác cao hơn kết quả phân loại được thực hiện bằng phương pháp phân loại ảnh có kiểm định truyền thống. $O$ 


\section{Tài liệu tham khảo}

[1]. Bateson, C. A., Asner, G. P., và Wessman, C. A. 2000. "Endmember bundles: A new approach to incorporating endmember variability into spectral mixture analysis". IEEE Trans. Geosci. Remote Sensing, Số. 38, trang. 1083-1094.

[2]. Bianchi. R., Cavalli. R., Fiumi. L. 2001. "CNR LARA project, Italy: Airborne laboratory for environmental research". Summaries of the V JPL Airborne Earth ScienceWorkshop, Pasadena, CA.

[3]. Baret, F., Guyot, G. 1991. Potentials and limit of vegetation indices for $\mathrm{LAl}$ and APAR assessment, Remote Sensing of Environment, Số 35, trang 161-173.

[4]. Bezdek, J. và Full, W. 1984. "FCM; The fuzzy c-means clustering algorithm", Computer and Geosciences, Số 10, trang 191-203.

[5]. Bosdogianni, P và Kittle, J. 1997. "Robus unmixing of large sets of mixed pixel”, Pattern Recognition Letters, Số 18(5), trang 415-424

[6]. Boardman, J. W. và Kruse, F. A. 1994. "Automated spectral analysis: A geological example using AVIRIS data, Northern Grapevine Mountains, Nevada," in Proc. 10th Thematic Conference, Geologic Remote Sensing, San Antonio.

[7]. Boardman, W., Kruse, F. A. và Green, R. O. 1995. "Mapping target signatures via partial unmixing of AVIRIS data". Summaries of the $V$ JPLAirborne Earth Science Workshop, Pasadena, CA.

[8]. Fisher, P. 1997. "The pixel: a snare and a delusion", International Journal of Remote Sensing, Số 18(3).

[9]. Foschi, G.P. 1994. A geometric approach to a mixed pixel problem: Detecting subpixel woody vegetation, Remote Sensing of Environment, Số
50(3), trang 317-327.

[10]. Jackson, R.D. 1983. Spectral Indices in n-Space, Remote Sensing of Environment, Số. 13, trang 409-421.

[11]. Floyd, F.S. 2013. Remote Sensing Principle and Interpretation.

[12]. Mao, C., Seal, M., và Heitschmidt, G. 1997. "Airborne hyperspectral image aquisition with digital CCD video camera". 16th Biennial Workshopon Videography and Color Photography in Resource Assessment, Weslaco, TX, trang 129-140.

[13]. Nageswara Rao, P.P. and Rao, V.R. 1987. Rice crop identification and area estimation using remotely-sensed data from Indian cropping patterns. International Journal of Remote Sensing, Số 8, trang 639-650.

[14]. Okamoto, K. and Fukuhara, M.1996. Estimation of paddy field area using the area ratio of categories in each mixel of Landsat TM. International Journal of Remote Sensing, Số 17, trang. 17351749.

[15]. Qi, J., Chehbouni, A., Heute, A.R., Kerr, Y.H. 1994. Modified Soil Adjusted Vegetation Index (MSAVI), Remote Sensing of Environment, Số. 48, trang 119-126.

[16]. Petrou, M. và Foschi, P. G. 1999. "Confidence in linear spectral unmixing of single pixels". IEEE Trans. Geosci. Remote Sensing, Số. 37, trang. 624-626.

[17]. Richardson, A.J. and Wiegand, C.L. 1977. Distinguishing vegetation from soil background information, Photogrammetric Engineering and Remote Sensing, Số. 43, trang 1541-1552.

[18]. R. O. Green và ctv.1998. "Imaging spectroscopy and the airborne visible/infrared imaging spectrometer (AVIRIS)". Remote Sens. Environ., Số. 65, 
trang. 227-248, 1998.

[19]. Settle, J. 1996. "On the relationship between spectral unmixing and subspace projection". IEEE Trans. Geosci. Remote Sensing, Số. 34, trang.1045-1046.

[20]. Shimabukuro, Y.E và Smith, J.A. 1991. "The least squares unmixing models to generate fraction images derived from remote sensing multispectral data", IEEE Transactions on Geoscience and Remote Sensing, Số 29(1), trang 16-20.

[21]. Short, M.N. và ctv. The remote sensing tutorial. NASA/GSFC. http://rst.gsfc.nasa.gov.

[22]. Tadjudin. S. và Landgrebe. D., 1998. "Classification of high dimensional data with limited training samples". Ph.D. dissertation, School of Elect. Eng. Comput. Sci., Purdue Univ., Lafayette, IN.

[23]. Tennakoon, S.B., Murty, V.V.N., and Eiumnoh, A. 1992. Estimation of cropped area and grain yield of rice using remote sensing data. International Journal of Remote Sensing, Số 13, trang.427-439.

[24]. Tuekey, C.J, 1979. Red and photographic infrared linear combination for monitoring vegetation. Remote Sensing, Số 8, trang 127-15.

[25]. Yamagata, Y., Wiegand, C., Akiyama, T., Shibayama, M. 1988. Water turbidity and perpendicular vegetation indices for paddy rice flood damage analyses, Remote Sensing of Environment Environment, Số. 26, Trang 241-251.O

\section{Summary}

The development of an approach for enhancing satellite image classification accuracy applied to mixed land surface - Experiment Section

Pham Minh Hai, Vietnam Institute of Geodesy and Cartography

Satellites imagery applications nowadays, serving the purposes of research, experiment or social life in general and in the field of Environment in particular, are increasingly popular. The two commonly used processes of extracting information are unsupervised and supervised classification. Supervised classification is the process of clustering pixels into classes based on training data (groups of pixels that represent areas) that you define. In supervised classification, a user can select sample pixels in an image that are representative of specific classes and then direct the image processing software to use these training sites (testing sets or input classes) as references for the classification of all other pixels in the image. However, when performing supervised classification in the area with mixed land surface, the lower accuracy of image classification is attributed to the effect of mixed pixels. Mixed pixel is a phenomenon that occurs when pixels with values belonging to class $A$, but are classified in other classes instead of class $A$. In data applications using small and medium-resolution, surface patches imaged as an individual pixels may contain more than one cover-type. As a result, mixed pixel classification by handling some components, including soil, water, vegetation on each pixel to improve the accuracy of classification results, is an important issue. $\bigcirc$ 